\title{
Article \\ Application of a Novel Attachable Magnetic Nerve Stimulating Probe in Intraoperative Lumbar Pedicle Screw Placement: A Porcine Model Study
}

\author{
Tae Sik Goh ${ }^{1} \mathbb{D}$, Sung-Chan Shin ${ }^{2}$, Hyun-Keun Kwon ${ }^{2}$, Eui-Suk Sung ${ }^{3}$, Se Bin Jun ${ }^{1}$, Byung-Joo Lee ${ }^{2, *},+\mathbb{D}^{\circ}$ \\ and Jung Sub Lee ${ }^{1, *,+}$
}

1 Department of Orthopaedic Surgery, Biomedical Research Institute, Pusan National University Hospital, Busan 49241, Korea; taesikgoh@gmail.com (T.S.G.); jun90815@naver.com (S.B.J.)

2 Department of Otorhinolaryngology-Head and Neck Surgery, Biomedical Research Institute, Pusan National University School of Medicine, Pusan National University, Pusan National University Hospital, Busan 49241, Korea; cha-nwi@hanmail.net (S.-C.S.); kwon-h-g@hanmail.net (H.-K.K.)

3 Department of Otolaryngology-Head and Neck Surgery, Research Institute for Convergence of Biomedical Science and Technology, College of Medicine, Pusan National University, Yangsan 46241, Korea; ch4oh@hanmail.net

* Correspondence: voiceleebj@gmail.com (B.-J.L.); jungsublee@pusan.ac.kr (J.S.L.); Tel.: +82-51-240-7335 (B.-J.L.); +82-51-240-7982 (J.S.L.); Fax: +82-51-246-8668 (B.-J.L.); +82-51-247-8395 (J.S.L.)

+ These authors contributed equally to this study.

check for updates

Citation: Goh, T.S.; Shin, S.-C.; Kwon, H.-K.; Sung, E.-S.; Jun, S.B.; Lee, B.-J.; Lee, J.S. Application of a Novel Attachable Magnetic Nerve Stimulating Probe in Intraoperative Lumbar Pedicle Screw Placement: A Porcine Model Study. Appl. Sci. 2021, 11, 7801. https://doi.org/10.3390/ app11177801

Academic Editor: Arkady Voloshin

Received: 16 July 2021

Accepted: 23 August 2021

Published: 25 August 2021

Publisher's Note: MDPI stays neutral with regard to jurisdictional claims in published maps and institutional affiliations.

Copyright: (c) 2021 by the authors. Licensee MDPI, Basel, Switzerland. This article is an open access article distributed under the terms and conditions of the Creative Commons Attribution (CC BY) license (https:/ / creativecommons.org/licenses/by/ $4.0 /)$.

\begin{abstract}
Pedicle screw instrumentation is a fundamental technique in lumbar spine surgery. However, several complications could occur when placing a pedicle screw, the most serious being damage to the neural structures. We developed an attachable magnetic nerve stimulating probe used for triggered electromyography (t-EMG) to avoid these. This study aimed to investigate the efficacy of this probe for intraoperative neuromonitoring (ION) during lumbar pedicle screw placement in a porcine model. Forty pedicle screws were inserted bilaterally into the pedicles of the fourth and fifth lumbar vertebrae of five pigs; 20 were inserted typically into the pedicle without nerve damage (Group A), and the other 20 were inserted through the broken medial wall of the pedicle to permit contact with the neural structures (Group B). We measured the triggered threshold for pedicle screw placement through the conventional nerve probe and our newly developed magnetic probe. There was no significant difference in the triggered threshold between the two instruments $(p=0.828)$. Our newly developed magnetic stimulating probe can be attached to a screwdriver, thus preventing real-time screw malpositioning and making it practical and equally safe. This probe could become indispensable in revision spine surgeries with severe adhesions or endoscopic spine surgeries.
\end{abstract}

Keywords: intraoperative neuromonitoring; magnetic nerve stimulating probe; pedicle screw

\section{Introduction}

Pedicle screws are essential for posterior fusion operations in lumbar degenerative diseases such as spinal stenosis and lumbar degenerative kyphosis [1,2]. However, instrumentation of pedicle screws is a relatively unsafe procedure and may cause complications such as dural tears, nerve root injury, and vascular injury [3-5]. In particular, care should be taken while inserting the pedicle screw since damage to the spinal cord or nerve root is most likely irreversible and could be fatal. Surgical expertise allows pedicle screw instrumentation to be executed uneventfully, but in the hands of inexperienced surgeons, it may result in a high incidence of neurological complications (0.8-6.1\%) due to screw malpositioning and pedicle breakage [6]. As safety is the topmost priority for both surgeons and patients, various surveillance devices, including image guidance systems and electromyographic tests for use during the actual surgery, are increasing in demand, and the interest in research in the field of spine surgery is on the rise. The most widely used surveillance 
devices during spinal surgery are image guidance systems, namely the dual-planar 2-D fluoroscopy and intraoperative 3-D scan $[7,8]$. However, the disadvantage of these imaging devices is that they do not offer real-time and direct monitoring of the procedure for nerve damage while the pedicle screws are being inserted. Therefore, in order to minimize the chances of complications and produce the optimal outcome for the patient, intraoperative fluoroscopy alone is inadequate and intraoperative neurophysiologic monitoring (ION) should be performed routinely.

Since 1992, when Calancie et al. introduced and proposed triggered electromyography (t-EMG) as a real-time ION technique during pedicle screw instrumentation, t-EMG has become increasingly popular for detecting intraoperative neural injuries in the field of spinal surgery $[9,10]$. A study by Lenke et al. demonstrated that a breach of the pedicle wall determines a significant difference in the threshold stimulus intensity through porcine experimental model research and prospective human study [11]. Additionally, several studies have proven that triggered EMG is useful for determining whether or not a pedicle screw has been inserted accurately [12-14].

In order to conduct ION during spinal surgery, nerve stimulating probes are required. A conventional probe has the disadvantage that it lacks the ability to detect intraoperative neural injury instantly. In addition, conventional probes and surgical instruments cannot be used simultaneously, and it is an inconvenience to replace the surgical instruments whenever ION is needed. To overcome these drawbacks of conventional probes, we develop novel magnetic nerve stimulating probes. The advantage of these probes is that the results of EMG can be known in real-time during the operation, which allows the immediate surgical application. In addition, they are convenient as they can be attached to all surgical instruments using magnetism.

Moreover, the probe developed by us has already demonstrated its efficacy and safety in thyroid and ear surgery, respectively $[15,16]$. Thus, we decided to use this newly developed probe in spinal surgeries where immediate recognition of nerve injury is vital. Therefore, we aimed to investigate and compare the efficacy of the novel magnetic stimulating probe and the conventional probe for ION during lumbar pedicle screw placement using a porcine model.

\section{Materials and Methods}

\subsection{Animal Experiment}

The protocol of the experiment was permitted by the Institutional Animal Care and Use Committee of Pusan National University, Yangsan Hospital (2019-015-A1C0(0)). The experiments were conducted on five adult female pigs. The pigs were sedated with an intramuscular injection of ketamine hydrochloride $(10 \mathrm{mg} / \mathrm{kg})$ and xylazine $(2 \mathrm{mg} / \mathrm{kg})$. Anesthesia was continued with inhalation of $3 \%$ isoflurane. The neuromuscular blockage was not used at any moment during the procedure. After completion of the experiment, potassium chloride was intravenously administered for euthanizing the pigs. One of the authors attached pairs of monopolar needle electrodes of the EMG bilaterally to the muscle bellies of the quadriceps, tibialis anterior, medial hamstring, and medial gastrocnemius, each $3 \mathrm{~cm}$ apart. A ground plate was attached to the skin between the stimulating and recording sites. A conventional stimulator (Direct nerve probe $100 \mathrm{~mm}$, Medtronic Xomed, Jacksonville, FL, USA) and the novel magnetic stimulator were used for the nerve stimulating cathode on pedicle screws (Figure 1). We developed the magnetic stimulator by taking the wire through which the conventional probe's current flows and replacing only the stimulator part with a magnet that could be attached to other surgical instruments (Figure 2). A nerve integrity monitor (NIM-Response 3.0 system: Medtronic Xomed, Jacksonville, FL, USA) was used to stimulate and record the triggered EMG. A constant current stimulation was delivered, and the intensity of the current was gradually increased $(0.1-80.0 \mathrm{~mA})$ until a reaction was obtained. The responses were recorded using a $10-20 \mathrm{kHz}$ filter setting, an amplifier sensitivity of $50 \mu \mathrm{V}$ per division, and a $50 \mathrm{~ms}$ time base. 


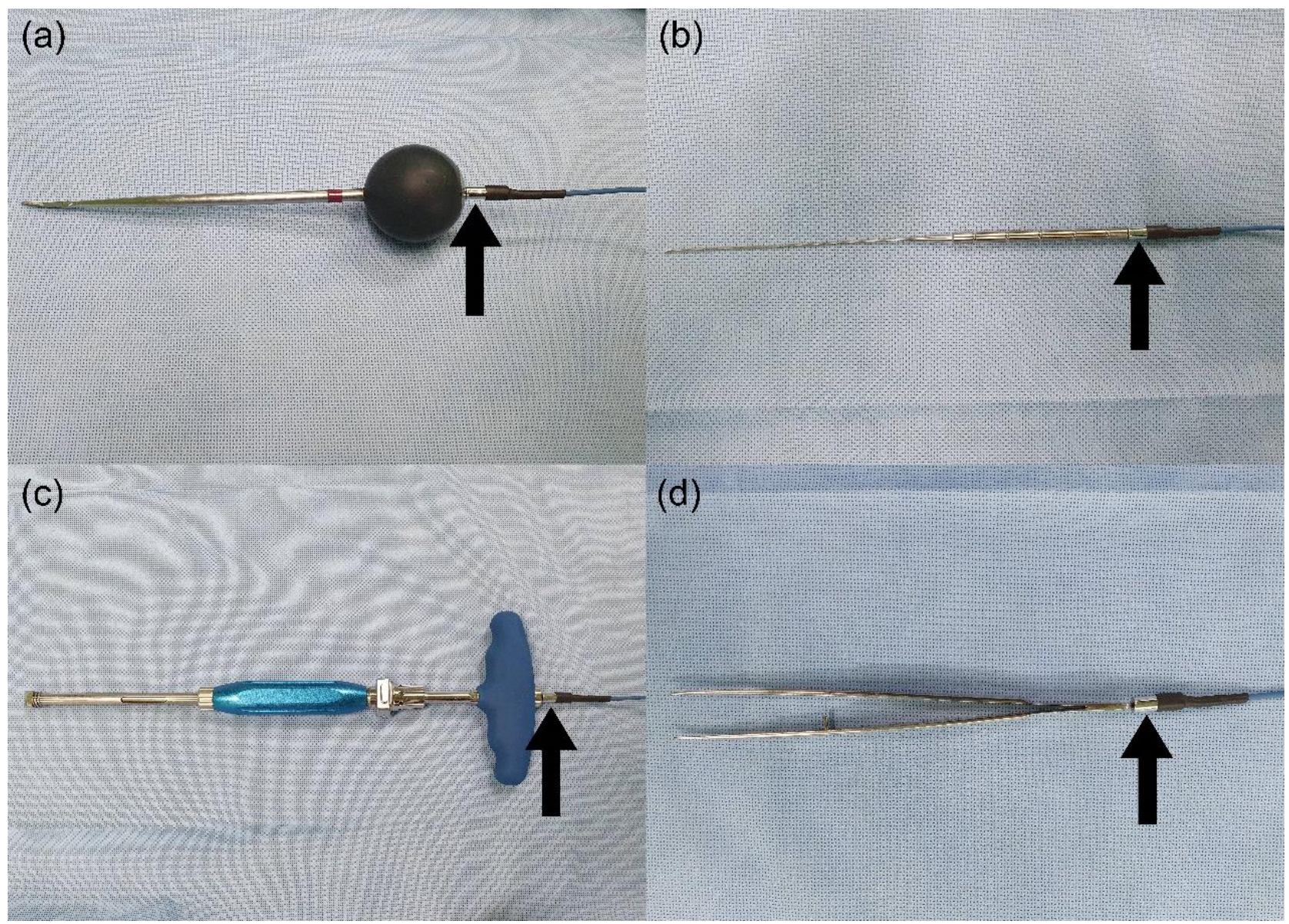

Figure 1. Magnetic nerve stimulating probe attached with various surgical instruments. The black arrow points to the magnet of the magnetic probe. (a) Gearshift probe. (b) Ball-tipped probe. (c) Pedicle screwdriver. (d) Long forcep.

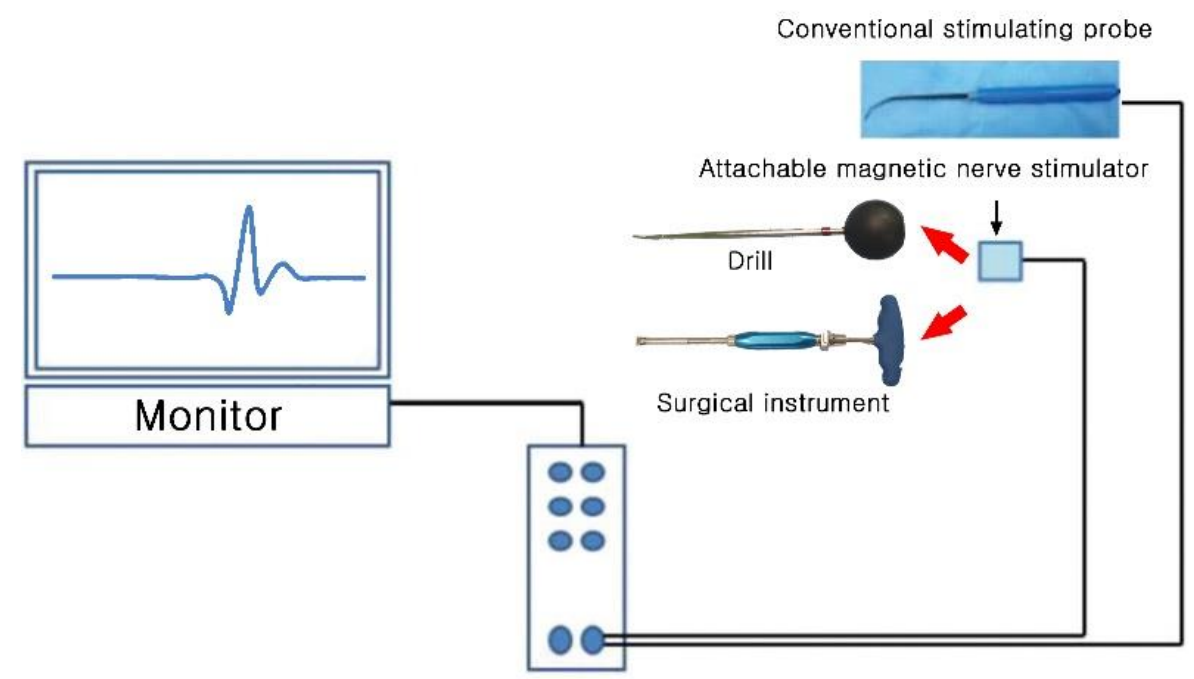

Figure 2. Schematic diagram comparing conventional probe and novel magnetic probe. The conventional probe is integrated with the wire, whereas the magnetic probe has the stimulator part replaced with a magnet and could be attached to other surgical instruments. In contrast, the wire carrying the electrical signal is the same for both probes.

A subperiosteal approach was adopted posteriorly until the lamina, facet joint, and transverse processes on both sides of the fourth and fifth lumbar vertebrae were exposed. Pedicle screws $(5.5 \times 35 \mathrm{~mm})$ were usually inserted (Group A, 20 pedicle screws) bilaterally 
into the pedicles of the fourth and fifth lumbar vertebrae by using an anatomical landmark (Figures 3 and 4). We performed the pedicle screw fixation in five steps. The first step is to find the point where the horizontal line that bisects the transverse process and the vertical line passing the lateral border of the superior articular facet meet and make an entry using a high-speed burr. The second step is to make a path from the posterior element through the pedicle to the anterior body using a gearshift probe through the created entry. Then, using a ball-tipped probe (Figure $1 b$ ), check whether the pedicle wall is intact. If it is intact, it works to widen the path created by using the tapper. Next, determine the length of the screw to be fixed while finally checking the integrity of the pedicle wall using a ball-tipped probe. Finally, we fix the pedicle screws. After fixing the screw, we experimented with measuring the trigger threshold while attaching the magnetic probe to the screwdriver and contacting the conventional probe to the driver tip to where the magnet was attached, as shown in Figure 1c. The screws were stimulated using our novel magnetic stimulating probe and the conventional probe, respectively, increasing the current until a t-EMG reaction was observed in the peripheral muscles. The minimum current (triggered threshold) that provoked a consistent and repetitive response was recorded. After completing the experiment, the pedicle screws that were usually inserted (Group A) were removed. The pedicle screws $(5.5 \times 35 \mathrm{~mm})$ were then abnormally inserted (Group B, 20 pedicle screws) along the path of the broken medial wall of the pedicle to allow the screw's propinquity to the neural complex (Figure 4). The broken pedicle walls and malpositioning of the pedicle screws were confirmed using the tactile sense of probing and by obtaining visual confirmation through a partial laminectomy (Figure 5). As in Group A, the experiments and recordings were conducted repeatedly in Group B.
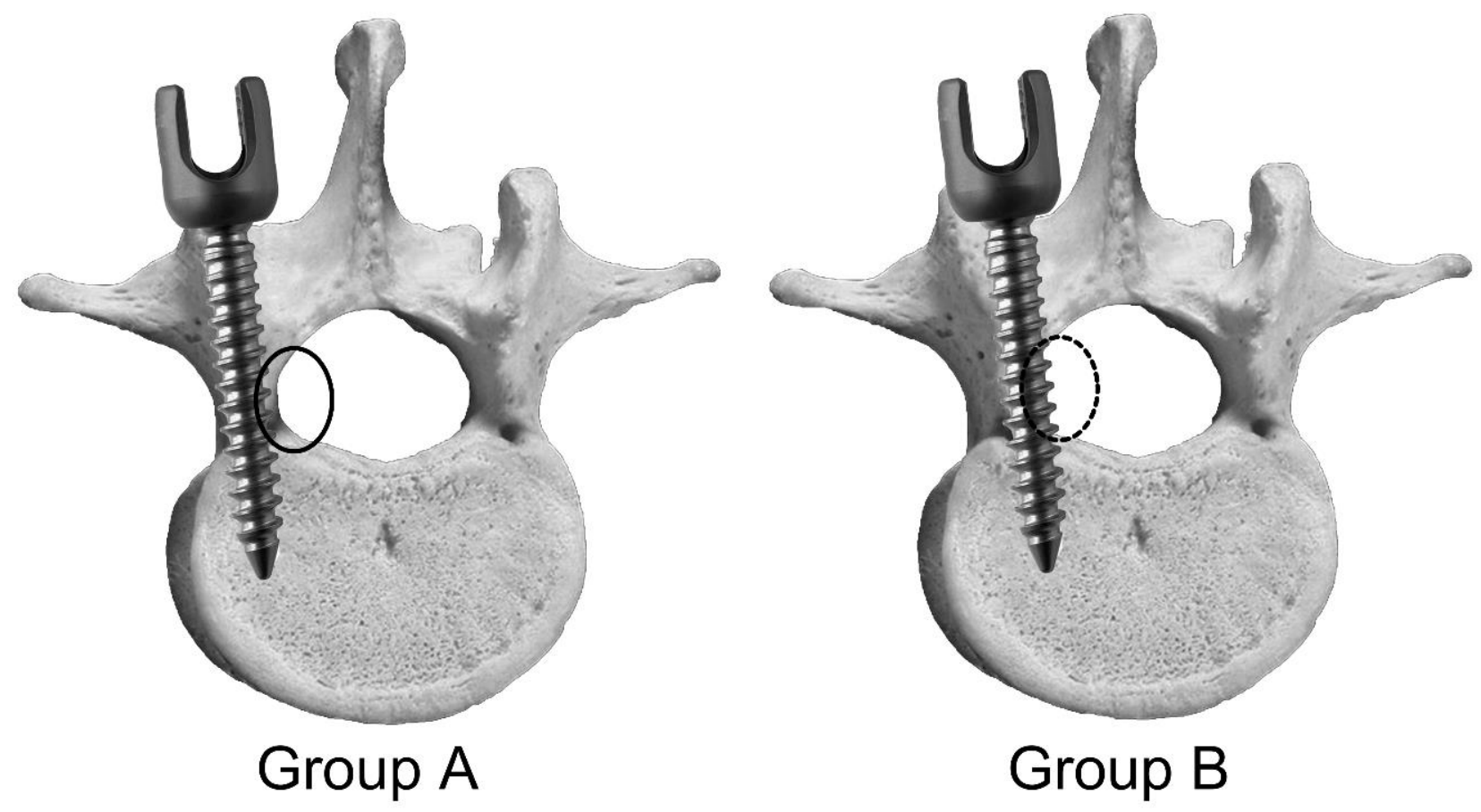

Figure 3. Schematic diagram of pedicle screw placement. Group A-pedicle screw insertion with intact pedicle wall; Group B-pedicle screw insertion through breached medial wall of pedicle. The solid black line shows that the medial wall of the pedicle is intact, and the black dotted line indicates that the broken medial wall of the pedicle and the pedicle screw is near the neural complex or dura through to the broken pedicle wall. 
(a)

(b)

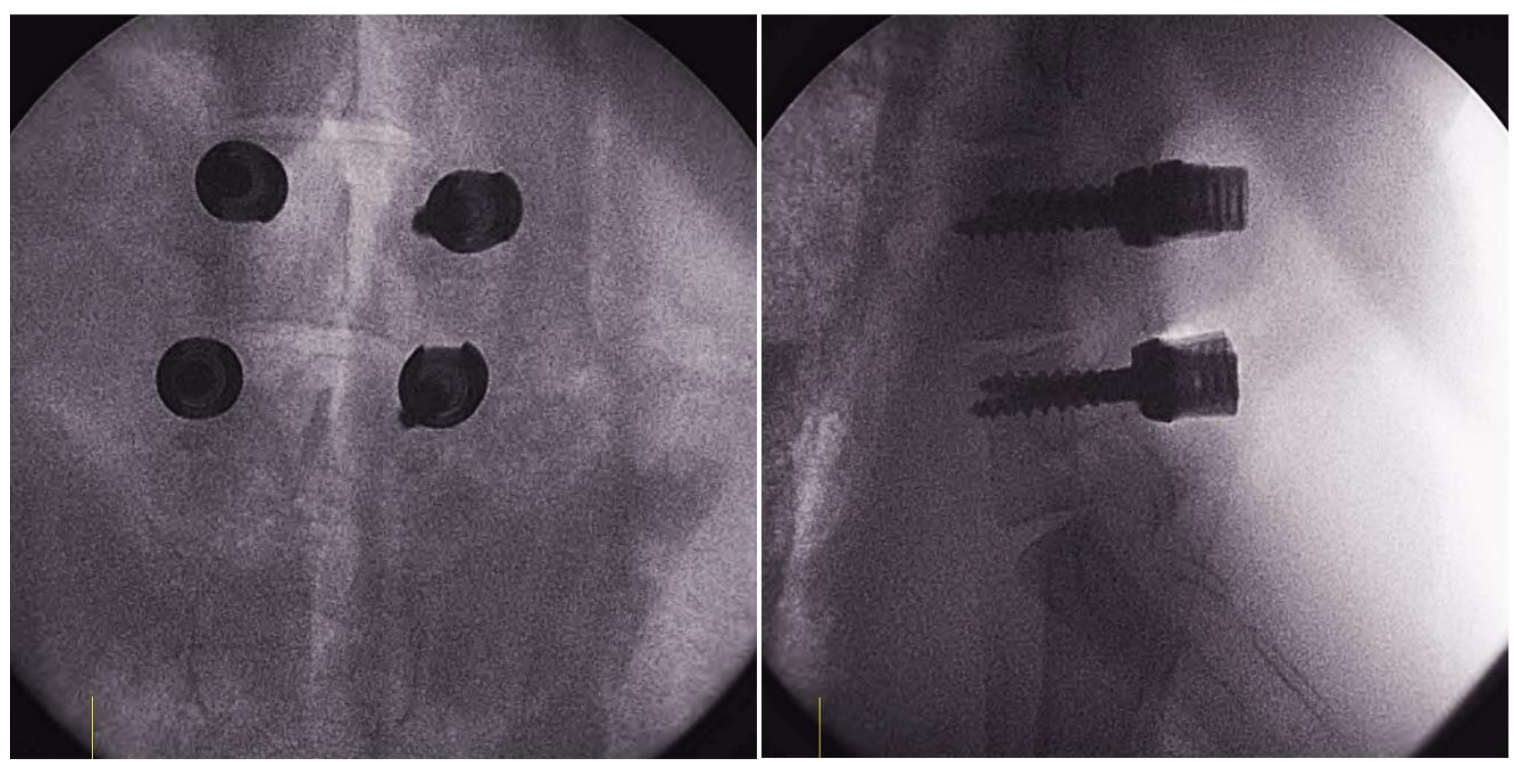

Figure 4. Intraoperative fluoroscopy. (a) Posteroanterior view. (b) Lateral view.

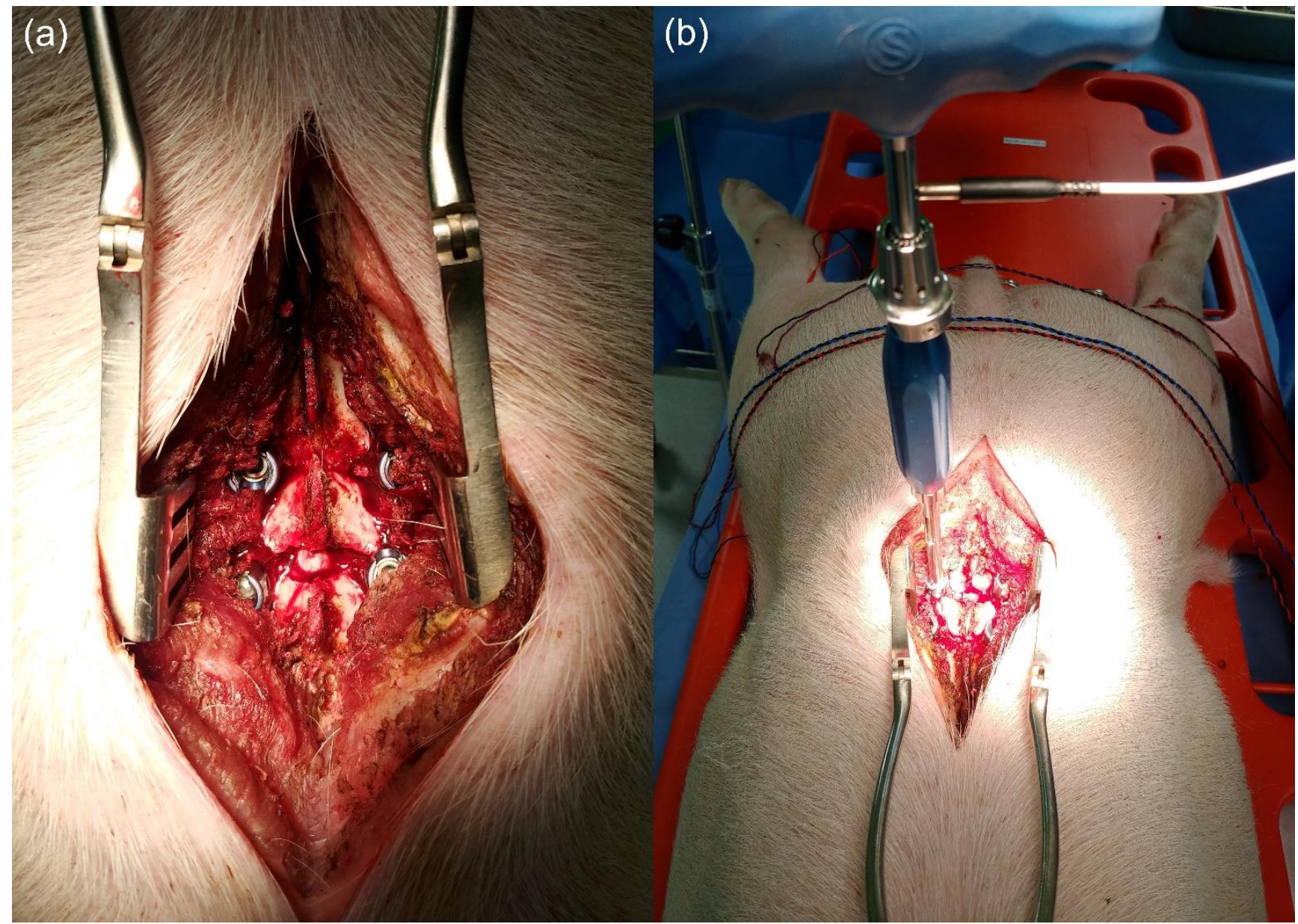

Figure 5. Intraoperative photographs. (a) Four inserted pedicle screws at pedicles of 4th and 5th lumbar vertebrae. (b) Triggered electromyography setting using magnetic nerve stimulator. 


\subsection{Statistical Analyses}

The statistical analyses were performed using the Mann-Whitney test with MedCalc for Windows, version 19.2 (MedCalc Software, Ostend, Belgium). A $p$ value less than 0.05 was considered statistically significant.

\section{Results}

A total of 40 pedicle screws were stimulated using a conventional probe and a newly developed magnetic stimulating probe, respectively, and the triggered threshold was measured. The raw data measured in this experiment are summarized in Table 1. The triggered threshold obtained from the usually inserted pedicle screws (Group A) showed no statistical difference between the conventional probe and the magnetic stimulating probe $(p=0.702$, Figure 6$)$. Similarly, there was no significant difference between the two groups from the experiment on Group B assuming neural injury during lumbar pedicle screw placement $(p=0.816$, Figure 6). Therefore, there was no difference in the triggered threshold between the two groups in the overall pedicle screw stimulation, including Groups A and B ( $p=0.828$, Figure 6). In addition, there were no differences between the two groups in the subgroup analysis based on the pedicle level and laterality (Figure 6). No complications or unexpected events were observed during the experiment when using a magnetic stimulating probe, and the results were comparable to those obtained using a conventional stimulating probe. The process of separating the driver after the instrumentation of the pedicle screw could be omitted in the magnetic stimulating probe. Thus, there was less hassle than in the conventional probe.

Table 1. Raw data of triggered threshold according to the use of conventional stimulator and novel magnetic stimulator.

\begin{tabular}{|c|c|c|c|c|c|c|}
\hline & & & \multicolumn{2}{|c|}{ Group A (Normal) } & \multicolumn{2}{|c|}{ Group B (Abnormal) } \\
\hline \multicolumn{3}{|c|}{ Triggered Threshold (mA) } & $\begin{array}{l}\text { Conventional } \\
\text { Stimulator }\end{array}$ & $\begin{array}{l}\text { Magnetic } \\
\text { Stimulator }\end{array}$ & $\begin{array}{l}\text { Conventional } \\
\text { Stimulator }\end{array}$ & $\begin{array}{l}\text { Magnetic } \\
\text { Stimulator }\end{array}$ \\
\hline \multirow{4}{*}{ Pig 1} & \multirow{2}{*}{$\mathrm{L} 4$} & Rt. & 16 & 17 & 3.5 & 3.5 \\
\hline & & Lt. & 12 & 12 & 1.0 & 0.8 \\
\hline & \multirow{2}{*}{ L5 } & Rt. & 11 & 12 & 0.7 & 0.7 \\
\hline & & Lt. & 10 & 10 & 3.0 & 3.5 \\
\hline \multirow{4}{*}{ Pig 2} & \multirow{2}{*}{$\mathrm{L} 4$} & Rt. & 13 & 14 & 3.5 & 3.5 \\
\hline & & Lt. & 10 & 10 & 2.5 & 2.0 \\
\hline & \multirow{2}{*}{ L5 } & Rt. & 8 & 8 & 2.0 & 2.0 \\
\hline & & Lt. & 9 & 10 & 1.5 & 1.5 \\
\hline \multirow{4}{*}{ Pig 3} & \multirow{2}{*}{$\mathrm{L} 4$} & Rt. & 10 & 10 & 0.8 & 1.0 \\
\hline & & Lt. & 8 & 7 & 3.0 & 3.0 \\
\hline & \multirow{2}{*}{ L5 } & Rt. & 9 & 9 & 2.5 & 2.5 \\
\hline & & Lt. & 12 & 12 & 3.0 & 2.5 \\
\hline \multirow{4}{*}{ Pig 4} & \multirow{2}{*}{$\mathrm{L} 4$} & Rt. & 15 & 16 & 1.0 & 1.5 \\
\hline & & Lt. & 13 & 13 & 2.5 & 2.5 \\
\hline & \multirow{2}{*}{ L5 } & Rt. & 12 & 13 & 0.7 & 1.0 \\
\hline & & Lt. & 15 & 15 & 1.5 & 1.5 \\
\hline \multirow{4}{*}{ Pig 5} & \multirow{2}{*}{$\mathrm{L} 4$} & Rt. & 11 & 11 & 2.0 & 2.5 \\
\hline & & Lt. & 9 & 9 & 3.0 & 3.0 \\
\hline & \multirow[b]{2}{*}{ L5 } & Rt. & 11 & 12 & 3.0 & 3.5 \\
\hline & & Lt. & 10 & 10 & 3.0 & 3.0 \\
\hline & $n \pm$ & & $11.2 \pm 2.25$ & $11.5 \pm 2.56$ & $2.19 \pm 0.95$ & $2.25 \pm 0.94$ \\
\hline
\end{tabular}

SD: standard deviation; Rt.: right; Lt.: left. 

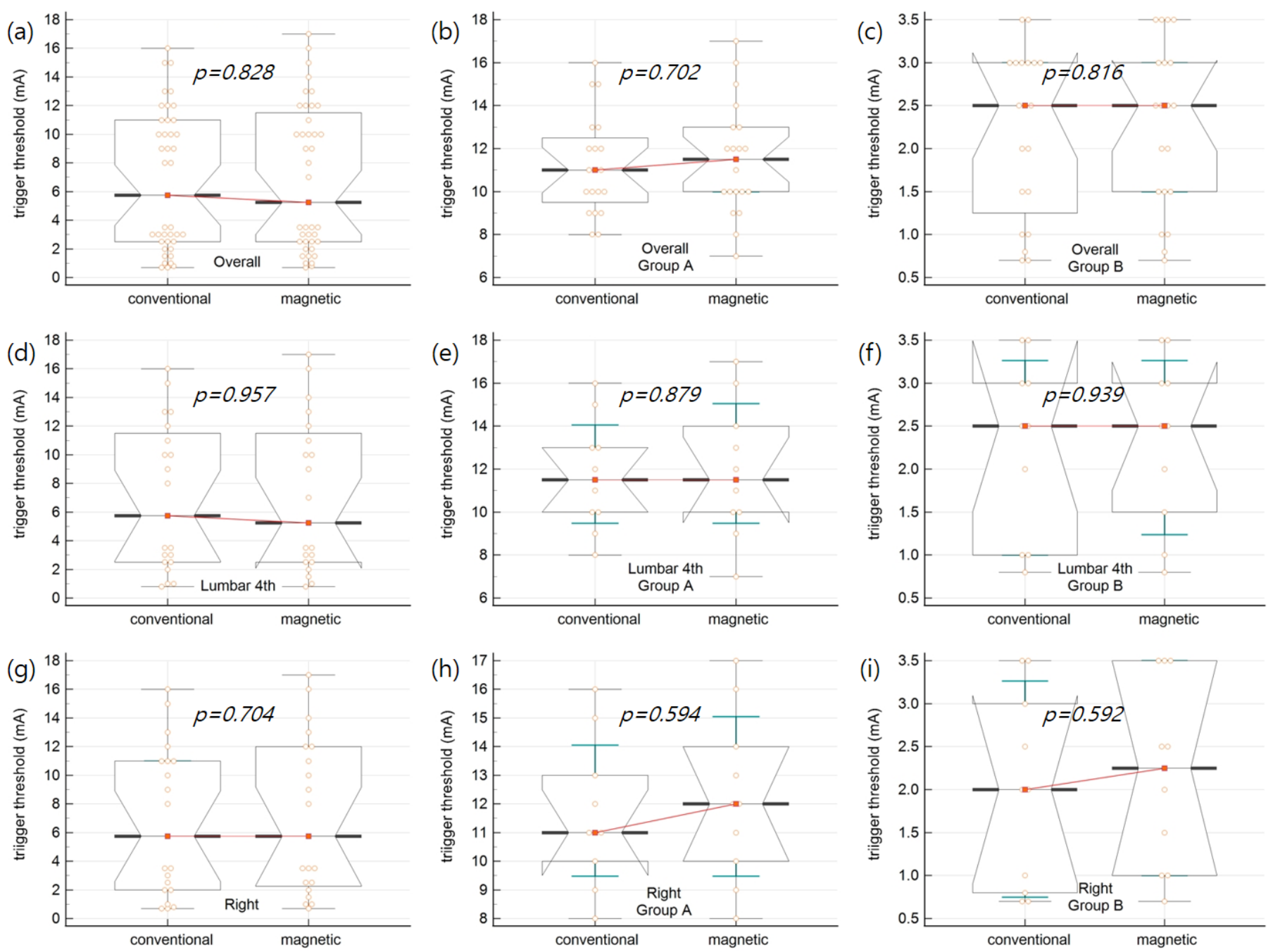

Figure 6. Comparison of triggered threshold according to the use of conventional stimulator and novel magnetic stimulator. Group A (intact pedicle wall), Group B (broken pedicle wall). (a-c) Overall, (a) total $(n=40)$, (b) Group A ( $n=20)$, (c) Group B $(n=20),(\mathbf{d}-\mathbf{f}) 4$ th lumbar vertebrae, (d) total $(n=20),(\mathbf{e})$ Group A $(n=10),(\mathbf{f})$ Group B $(n=10),(\mathbf{g}-\mathbf{i})$ Right side, (g) total $(n=20),(\mathbf{h})$ Group A $(n=10)$, (i) Group B $(n=10)$.

\section{Discussion}

This study found no significant difference in the triggered threshold between the conventional probe and the newly developed magnetic probe that can be attached to a screwdriver, thus preventing real-time screw malpositioning and making it hassle-free, practical, and equally safe. The reason that there was no difference in the triggered threshold is that the wire part through which the current of the two probes flows is the same as shown in Figure 2, and the tip of the conventional probe is placed on the part where the magnetic probe can be attached as shown in Figure 1c.

Calancie et al. demonstrated a pedicle screw threshold test (PSTT) in an experimental study using constant voltage stimulation in a porcine model. Triggered electromyography, has the advantage of allowing immediate assessment of the condition of the pedicle during surgery unlike other electrophysiological tests such as somatosensory evoked potentials and motor evoked potentials $[9,10,17]$. Moreover, triggered electromyography is sensitive to individual nerve root injury, making it easy to examine multiple sites concurrently $[9,17]$. Several studies also suggest that $\mathrm{t}$-EMG could help prevent pedicle screw malpositioning [18-20]. A meta-analysis study revealed that PSTT showed a weak diagnostic accuracy for pedicle screw malpositioning of the lumbar and thoracic spines [19]. However, when the triggered threshold during lumbar pedicle screw fixation was less than $8 \mathrm{~mA}$, the diag- 
nostic odds ratios for sensitivity and specificity were high, indicating that PSTT is suitable for predicting screw malpositioning during lumbar pedicle screw placement, which is consistent with our results [19].

In order to perform a t-EMG during lumbar pedicle screw placement, a probe that transmits a current stimulus to the pedicle screw is required. To overcome the shortcomings of conventional probes, we developed a novel magnetic nerve stimulating probe for spine surgery. This probe can be attached to all magnetic metallic surgical instruments (Figure 1). There was no significant difference in the triggered threshold of the conventional nerve stimulating probe and the novel magnetic nerve stimulating probe when both the pedicle walls were intact nor when the pedicle wall was breached during pedicle screw placement in the porcine model. We demonstrated that our novel nerve stimulating probe attached to a pedicle screwdriver could provide adequate information about the breaching of the wall and the proximity of the screw to the neural structures during pedicle screw placement. The results of our study were consistent with those of several earlier studies on trigger thresholds. According to a study published by Lenke et al., a pedicle screw with a threshold of $8 \mathrm{~mA}$ or more could be considered capable of being correctly inserted into the pedicle [11]. For a screw with a threshold of less than $8 \mathrm{~mA}$, pedicle screw malpositioning could be suspected. Furthermore, they reported that when the threshold is less than $4 \mathrm{~mA}$, the screw is inserted through the pedicle wall defect, and there is strong likelihood that the dura or nerve roots may be close to the screw [11]. As a result of our experiment, the threshold median value was more than $10 \mathrm{~mA}$ when the pedicle wall was intact, and the threshold median value was less than $3 \mathrm{~mA}$ when the pedicle wall was broken. Thus, our results are consistent with the previous studies [11,19].

The advantage of this novel device is that it offers real-time, hassle-free monitoring, thereby preventing pedicle screw malpositioning and nerve damage. When a pedicle screw is inserted using the freehand technique, the surgeon identifies the anatomical landmark and considers the transverse and sagittal pedicle angles. The surgeon then performs the five following steps: preparing a screw entry point, marking the screw trajectory using a pedicle finder or gear shift probe, palpating the trajectory to inspect the medial and inferior walls for breaches, tapping, and finally inserting the pedicle screw. The neural structures are exposed to potential damage during this five-step process, except while creating an entry. Utilizing a conventional nerve stimulating probe involves the hassle of removing the surgical instruments used at each step to insert the probes. As described above, because the neuromonitoring using conventional probes is performed after completing each step, it is difficult to view it as real-time monitoring as it would have the disadvantage with respect to time and the technical aspects. In contrast, the newly developed magnetic stimulating probe can easily be attached to and detached from the surgical instrument used at each step, thus making it is less cumbersome to replace. Moreover, it can be utilized as a real-time warning tool for pedicle screw malpositioning to avoid neural complex injury. However, if the surgical tool is rotated clockwise during tapping or screw fixing, the magnetic probe may fall off because it is only connected magnetically. To reiterate, if the pedicle is broken or nerve damage occurs, the conventional probe could check nerves only after each surgical step is completed, so it is impossible to know the nerve damage in real-time. However, our magnetic probe could prevent nerve damage in advance. It could serve as a real-time neuromonitoring system because it could attach to the surgical tool in every step. Therefore, this novel magnetic stimulating probe that can recognize pedicle wall breach or neural insult in real-time while placing a pedicle screw may be considered to be more valuable than a conventional stimulating probe.

Image-guided systems, i.e., 2D fluoroscopy and intraoperative 3D scanning, are the most widely used surveillance devices during spinal surgery $[7,8]$. Although they have the advantage of being easy to use, these imaging devices only provide information about the anatomical relationship between the pedicle screw and surrounding structures but do not directly inform whether or not neurological damage has occurred. In other words, it is not enough to infer that the nerves may have been injured by figuring out the 
abnormal screw position. Unlike these image-guided systems, the electrophysiology-based neural monitoring device is somewhat cumbersome but has the advantage of directly detecting neural damage, and no additional irradiation is required. As in this study, if a neural monitoring system capable of real-time monitoring that does not require additional procedures, effort, and time other than surgery is introduced in the near future, it could be a future-proof alternative for image-guided systems and is expected to help doctors with challenging surgery or less experience.

In addition, it is difficult or impossible to conduct ION with conventional probes in revision or endoscopic surgeries. However, using our novel stimulating probe could enable hassle-free and convenient ION during revision surgery or endoscopic spine surgery, in which it is challenging to identify anatomical structures. The distance and positional relationship between the neural tissue and the surgical instrument used could be verified and monitored in real-time without replacing the instrument or the probe. Additionally, it can be applied not only in lumbar spine surgeries, but also in all areas of spinal surgery such as cervical degenerative diseases and spinal deformities. Especially, it could make scoliosis surgery safer, which requires a high degree of proficiency in pedicle screw insertion. It could also be used for percutaneous pedicle screw placement, one of the main procedures in minimally invasive spinal surgery that has been widely practiced in recent years.

The disadvantage of this study was that it was conducted only in the porcine model. We have, therefore, planned to conduct a feasibility and safety test for using the magnetic nerve stimulating probe in human spinal surgery in the near future. If threshold t-EMG testing is constantly performed during spine surgeries, and if the threshold data obtained during each operation is accumulated and a standardized protocol is established through this, spine surgery could be safely performed, including pedicle screw insertion as a decompression procedure during spine surgery. In addition, the experiment was not conducted at various stages of pedicle instrumentation but was performed on an inserted pedicle screw. Another limitation is that the experiment was conducted only with a pedicle screwdriver, not with various tools. Additionally, the absence of amplitude values of t-EMG is considered a limitation of this study. Sung et al. applied an attachable nerve stimulator to various instruments used in endoscopic or robotic thyroidectomy and reported that there were no significant differences in stimulating EMG amplitude values compared to a conventional stimulator [21]. This study compensates to some extent the limitations of our study by showing similar results in several surgical instruments that may have different resistance values.

\section{Conclusions}

In summary, the threshold current did not show a statistical difference between the conventional nerve stimulating probe and the newly developed magnetic nerve stimulating probe. Our magnetic probe showed that the performance was not inferior to the conventional probe in terms of electroneurophysiology during pedicle screw placement but was simple and convenient to use. In addition, it enabled an irradiation-free and real-time intraoperative neuromonitoring system during pedicle screw placement.

Author Contributions: Conceptualization, J.S.L. and B.-J.L.; methodology, B.-J.L.; investigation, H.-K.K. and S.B.J.; writing-original draft preparation, T.S.G.; writing-review and editing, J.S.L.; supervision, B.-J.L.; project administration, S.-C.S. and E.-S.S.; funding acquisition, T.S.G. All authors have read and agreed to the published version of the manuscript.

Funding: This research was supported by Basic Science Research Program through the National Research Foundation of Korea (NRF) funded by the Ministry of Education (NRF-2018R1D1A1B07047666, TSG; NRF-2021R1F1A1064056, TSG) and the Korean Health Technology R\&D project (HI18C2383) through the Korea Health Industry Development Institute (KHIDI), funded by the Ministry of Health \& Welfare, Republic of Korea. 
Institutional Review Board Statement: The protocol of the experiment was permitted by the Institutional Animal Care and Use Committee of Pusan National University, Yangsan Hospital (2019-015$\mathrm{A} 1 \mathrm{C} 0(0))$

Informed Consent Statement: Not applicable.

Data Availability Statement: The raw data of the study are included in the results section of this paper.

Conflicts of Interest: The authors declare no conflict of interest.

\section{References}

1. Boachie-Adjei, O.; Girardi, F.P.; Bansal, M.; Rawlins, B.A. Safety and efficacy of pedicle screw placement for adult spinal deformity with a pedicle-probing conventional anatomic technique. J. Spinal Disord. 2000, 13, 496-500. [CrossRef] [PubMed]

2. Liljenqvist, U.R.; Halm, H.F.; Link, T.M. Pedicle screw instrumentation of the thoracic spine in idiopathic scoliosis. Spine (Phila Pa 1976) 1997, 22, 2239-2245. [CrossRef]

3. Kosmopoulos, V.; Theumann, N.; Binaghi, S.; Schizas, C. Observer reliability in evaluating pedicle screw placement using computed tomography. Int. Orthop. 2007, 31, 531-536. [CrossRef] [PubMed]

4. Nevzati, E.; Marbacher, S.; Soleman, J.; Perrig, W.N.; Diepers, M.; Khamis, A.; Fandino, J. Accuracy of pedicle screw placement in the thoracic and lumbosacral spine using a conventional intraoperative fluoroscopy-guided technique: A national neurosurgical education and training center analysis of 1236 consecutive screws. World Neurosurg. 2014, 82, 866-871.e2. [CrossRef]

5. Shi, Y.B.; Binette, M.; Martin, W.H.; Pearson, J.M.; Hart, R.A. Electrical stimulation for intraoperative evaluation of thoracic pedicle screw placement. Spine (Philipa Pa 1976) 2003, 28, 595-601. [CrossRef]

6. Esses, S.I.; Sachs, B.L.; Dreyzin, V. Complications associated with the technique of pedicle screw fixation. A selected survey of ABS members. Spine (Philipa Pa 1976) 1993, 18, 2231-2238. [CrossRef]

7. Dunn, C.; Faloon, M.; Milman, E.; Pourtaheri, S.; Sinah, K.; Hwang, K.; Emami, A. Accuracy and safety of percutaneous lumbosacral pedicle screw placement using dual-planar intraoperative fluoroscopy. Asian Spine J. 2018, 12, 238-245. [CrossRef]

8. Santos, E.R.; Ledonio, C.G.; Castro, C.A.; Trong, W.H.; Sembrano, J.N. The accuracy of intraoperative O-arm images for the assessment of pedicle screw postion. Spine (Philipa Pa 1976) 2012, 37, E119-E125. [CrossRef] [PubMed]

9. Calancie, B.; Lebwohl, N.; Madsen, P.; Klose, K.J. Intraoperative evoked EMG monitoring in an animal model. A new technique for evaluating pedicle screw placement. Spine (Philipa Pa 1976) 1992, 17, 1229-1235. [CrossRef] [PubMed]

10. Calancie, B.; Madsen, P.; Lebwohl, N. Stimulus-evoked EMG monitoring during transpedicular lumbosacral spine instrumentation. Initial clinical results. Spine (Philipa Pa 1976) 1994, 19, 2780-2786. [CrossRef] [PubMed]

11. Lenke, L.G.; Padberg, A.M.; Russo, M.H.; Bridewell, K.H.; Gelb, D.E. Triggered electromyographic threshold for accuracy of pedicle screw placement. An animal model and clinical correlation. Spine (Philipa Pa 1976) 1995, 20, 1585-1591. [CrossRef] [PubMed]

12. Lewis, S.J.; Lenke, L.G.; Raynor, B.; Long, J.; Bridwell, K.H.; Padberg, A. Triggered electromyographic threshold for accuracy of thoracic pedicle screw placement in a porcine model. Spine (Philipa Pa 1976) 2001, 26, 2485-2489. [CrossRef] [PubMed]

13. Raynor, B.L.; Lenke, L.G.; Bridwell, K.H.; Taylor, B.A.; Padberg, A.M. Correlation between low triggered electromyographic thresholds and lumbar pedicle screw malposition: Analysis of 4857 screws. Spine (Philipa Pa 1976) 2007, 32, 2673-2678. [CrossRef]

14. Raynor, B.L.; Lenke, L.G.; Kim, Y.; Hanson, D.S.; Wilson-Holden, T.J.; Bridwell, K.H.; Padberg, A.M. Can triggered electromyograph thresholds predict safe thoracic pedicle screw placement? Spine (Philipa Pa 1976) 2002, 27, 2030-2035. [CrossRef] [PubMed]

15. Oh, S.J.; Choi, S.; Lee, S.; Na, H.; Seung, E.; Shin, S.; Lee, B. Application of attachable magnetic nerve stimulator in intraoperative facial nerve monitoring during ear surgery. Otolaryngol. Head Neck Surg. 2020, 162, 773-775. [CrossRef]

16. Sung, E.S.; Lee, J.; Shin, S.; Chois, S.; Jung, D.; Lee, B. Development of a novel detachable magnetic nerve stimulator for intraoperative neuromonitoring. World J. Surg. 2018, 42, 137-142. [CrossRef]

17. Maguire, J.; Wallace, S.; Madiga, R.; Leppanen, R.; Draper, V. Evaluation of intrapedicular screw position using intraoperative evoked electromyography. Spine (Philipa Pa 1976) 1995, 20, 1068-1074. [CrossRef]

18. De Blas, G.; Barrios, C.; Regidor, I.; Montes, E.; Burgos, J.; Piza-Vallespir, G.; Hevia, E. Safe pedicle screw placement in thoracic scoliotic curves using t-EMG: Stimulation threshold variability at concavity and convexity in apex segments. Spine (Philipa Pa 1976) 2012, 37, E387-E395. [CrossRef]

19. Lee, C.; Kim, H.; Kim, H.R.; Lee, C.; Kim, J.; Sala, F. Can triggered electromyography thresholds assure accurate pedicle screw placements? A systematic review and meta-analysis of diagnostic test accuracy. Clin. Neurophysiol. 2015, 126, $2019-2025$. [CrossRef]

20. Shaw, K.A.; Murphy, J.S.; Devito, D.P. Accuracy of robot-assisted pedicle screw insertion in adolescent idiopathic scoliosis: Is triggered electromyographic pedicle screw stimulation necessary? J. Spine Surg. 2018, 4, 187-194. [CrossRef]

21. Sung, E.; Lee, J.; Kim, S.H.; Shin, S.; Jung, D.; Lee, B. Developmnet of an attachable endoscopic nerve stimulator for intraoperative neuromonitoring during endoscopic or robotic thyroidectomy. Otolaryngol. Head Neck Surg. 2018, 158, 465-468. [CrossRef] [PubMed] 